\title{
ISOMORPHISM TYPES OF INFINITE ALGEBRAS ${ }^{1}$
}

\section{S. D. COMER AND J. J. LE TOURNEAU}

A basic problem in the general theory of algebras is the determination of the number of nonisomorphic algebras with a given (finitary) similarity type and infinite power. Our main result is to show that in every case the number of such algebras actually equals the most naturally computed upper bound. In particular we show that if $\tau$ is any similarity type containing $\mathfrak{n}$ symbols, at least one of which is positive rank, and $\mathfrak{m}$ is any infinite cardinal then there exist exactly $2^{\mathfrak{m} \cdot \mathfrak{n}}$ nonisomorphic algebras with similarity type $\tau$ and power $\mathfrak{m}$. The corresponding problem for finite algebras was considered in Harrison [1].

In $\S 0$ we introduce definitions and accompanying notation; in $\S 1$ we consider the special problem of determining the number of nonisomorphic unary algebras (our work is based on the construction presented before Lemma 1); and in $\$ 2$ we consider this determination problem for arbitrary algebraic similarity types.

0 . We assume that each ordinal number is equal to the set of all smaller ordinal numbers and that each cardinal number is an initial ordinal. The function $\boldsymbol{\aleph}$ defined on all ordinals is the usual enumeration of the infinite cardinals. Ordinals are usually denoted by $\alpha, \beta$, $\gamma, \cdots$ and cardinals by $\mathfrak{m}, \mathfrak{n}, \cdots$. The smallest infinite cardinal $\boldsymbol{\aleph}_{0}$ is sometimes denoted by $\omega$. The cardinality (or power) of a set $A$ is denoted by $|A|$. A function $f$ on $A$ into $B$ is a function whose domain is $A$ and whose range is a subset of $B$; in symbols $D f=A$ and Rf $\subseteq B$. For any sets $A, B, A B$ is the set of all functions on $A$ into $B$. For any integer $n$ and any nonempty set $A, f$ is an $n$-ary operation on $A$ iff $f \in{ }^{n}{ }_{A} A$.

By a similarity type $\tau$ we mean any function on an ordinal into $\omega$. For any similarity type $\tau, \mathfrak{A}=\langle A, \mathcal{O}\rangle$ is a $\tau$-algebra or an algebra of type $\tau$ iff $\mathscr{A}$ is an ordered pair whose first coordinate (called the universe of $\mathfrak{A}$ ) is a nonempty set $A$ and whose second coordinate (called the operations of $\mathfrak{A}$ ) is a function $\mathcal{O}$ on $\mathbb{D} \tau$ such that, for any $\alpha \in \mathbb{D} \tau, \mathcal{O}_{\alpha}$ is a $\tau(\alpha)$-ary operation on $A$. The number $\tau(\alpha)$ is called the rank of $\hat{\theta}_{\alpha}$. If $\tau(\alpha)=0$ or $\tau(\alpha)=1$ we identify $\theta_{\alpha}$ in the natural way with a member of $A$ or a function on $A$ into $A$, respectively. If $\mathfrak{A}$ is an algebra

Received by the editors July 20, 1968 .

1 The work of the first author was supported in part by NSF Grant GP 7252. The authors wish to thank Professor J. Donald Monk for bringing this problem to their attention. 
whose only operation is of rank 1 then $\mathfrak{A}$ is called a unary algebra. Algebras are denoted by $\mathfrak{A}, \mathfrak{B}, \mathfrak{C}, \cdots$

1. In this section we prove that for any infinite cardinal $\mathfrak{m}$ there are exactly $2^{\mathfrak{m}}$ nonisomorphic root unary algebras of power $\mathfrak{m}$. By a root unary algebra we mean a unary algebra $\mathfrak{A}$ whose universe contains exactly one point which is sent to itself under the operation of $\mathfrak{A}$ and such that every subalgebra of $\mathfrak{A}$ also contains this "fixed point." The class of all root unary algebras (or roots) is denoted by $R$ and for any cardinal $\mathfrak{m}$ the class of all roots of power $\leqq \mathfrak{m}$ is denoted by $R_{\mathfrak{m}}$. The proof of the above claim rests on the construction outlined below.

For any infinite cardinal $\mathfrak{m}$, for any function $f$ on $\mathfrak{m}$ into $R$, and for any function $g$ on $m$ into $\{0,1\}$, let $\mathfrak{S}(f, g)$ be the root pictured in Figure 1. To construct $\mathfrak{S}(f, g)$ set-theoretically first form disjoint isomorphic copies of all members in the range of $f$. These copies are then combined into a single structure by mapping each of the old fixed points, not to themselves, but to a new point, each of these new

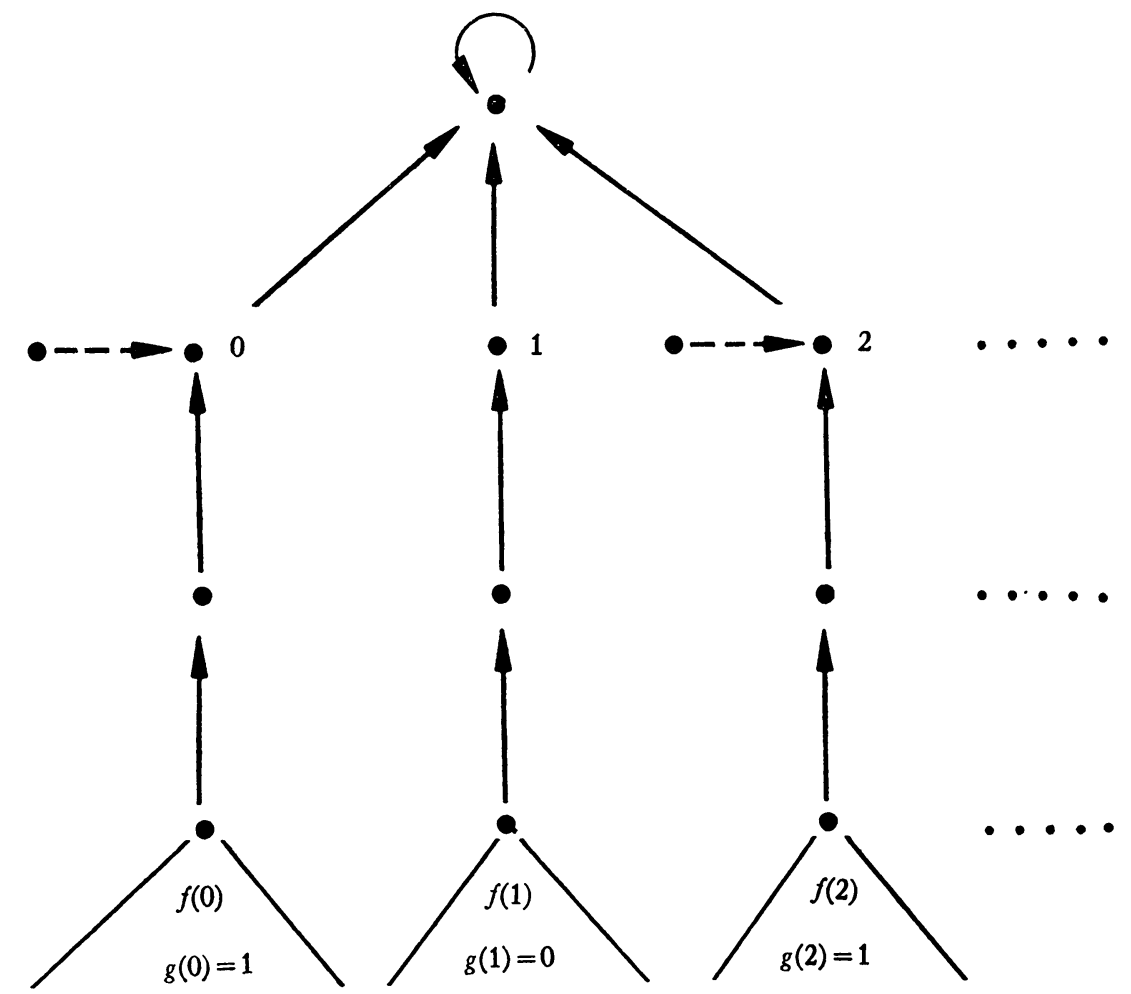

Figure 1 
points to a second new point, and finally all of these last points to a single fixed point (see Figure 1). With each point $p$ in the above construction such that the image of $p$ is the new fixed point there is a naturally associated ordinal in $\mathrm{m}$. As the final step of our construction we tie a new point to each of these layer one points whose associated ordinal has the value 1 under the function $g$ (see dotted arrows in Figure 1).

LEMma 1. For any infinite cardinal $\mathfrak{m}$, for any function $f$ on $\mathfrak{m}$ into $R$, and for any functions $g_{1}$ and $g_{2}$ on $\mathfrak{m}$ into $\{0,1\}$ :

(1) If $\operatorname{Rf} \subseteq R_{\mathfrak{m}}$ then $\mathbb{S}\left(f, g_{1}\right) \in R_{\mathfrak{m}}$.

(2) If no two members of Rf are isomorphic then $\mathbb{E}\left(f, g_{1}\right)$ is isomorphic to $\mathbb{S}\left(f, g_{2}\right)$ iff $g_{1}=g_{2}$.

Proof. (1) $\mathfrak{S}\left(f, g_{1}\right)$ is clearly a member of $R$ and consists of $m$ parts, each of which has cardinality $\leqq \mathfrak{m}$. Thus there are less than or equal $\mathfrak{m}^{2}=\mathfrak{m}$ points in the universe of $\mathfrak{E}\left(f, g_{1}\right)$, which implies $\mathfrak{E}\left(f, g_{1}\right) \in R_{\mathfrak{m}}$.

(2) If $\mathfrak{S}\left(f, g_{1}\right)$ is isomorphic to $\mathfrak{S}\left(f, g_{2}\right)$ by $h$, then for any $\mathfrak{A}$ in $\Re f$ there is a $\mathfrak{B}$ in $\mathscr{R} f$ such that the isomorphism $h$ induces an isomorphism between $\mathfrak{A}$ and $\mathfrak{B}$. But by the assumption concerning $\mathscr{R} f$ this implies that $h$ is the identity function, which in turn implies that $g_{1}=g_{2}$.

THEOREM 1. For any infinite cardinal $\mathfrak{m}$ there are exactly $2^{\mathfrak{m}}$ nonisomorphic unary algebras with power $\mathrm{m}$.

Proof. Let $A$ be a set of cardinality m. Since every member of ${ }^{A} A$ can be identified with a subset of ordered pairs of elements in $A$ it follows that there are at most $2^{\mathfrak{m} \cdot \mathfrak{m}}=2^{\mathfrak{m}}$ different algebras whose universe is $A$. Thus there are at most $2^{\mathfrak{m}}$ nonisomorphic algebras of power $\mathrm{m}$.

In order to prove that equality holds we define by induction on ordinals a collection $K_{\alpha}$ of sets of roots such that for every ordinal $\alpha, K_{\alpha}$ contains $2^{\aleph_{\alpha}}$ pairwise nonisomorphic roots each of which has power $\boldsymbol{\aleph}_{\alpha}$. The proof of these facts follows by a straightforward induction using the definition of $K_{\alpha}$, Lemma 1, and the fact that for any infinite cardinal $\mathfrak{m}$ there are $2^{\mathfrak{m}}$ functions in $\mathfrak{m}\{0,1\}$. Speaking vaguely, each member of $K_{\alpha}$ "codes-in" a different member of $\aleph_{\alpha}\{0,1\}$.

For any $n \in \omega$ let $\mathfrak{A}_{n}$ be the root whose universe is $\{0, \cdots, n\}$, whose fixed point is 0 , and such that every nonzero element of the universe of $\mathfrak{A}_{n}$ is sent to its predecessor by the operation of $\mathfrak{A}_{n}$. Let $K=\left\{\mathfrak{A}_{n}: n \in \omega\right\}$. Note that there are $\boldsymbol{\aleph}_{0}$ nonisomorphic members of $K$. Let $f_{0}$ be a 1-1 function on $\aleph_{0}$ into $K$. 
(a) $K_{0}=\left\{\mathfrak{S}\left(f_{0}, g\right): g \in^{\mathbb{N}_{0}}\{0,1\}\right\}$.

(b) Assume $K_{\alpha}$ is well defined. Let $f_{\alpha+1}$ be a 1-1 function on $\boldsymbol{\aleph}_{\alpha+1}$ into $K_{\alpha}$ (from the induction hypothesis $\left|K_{\alpha}\right|=2^{\aleph_{\alpha}} \geqq \boldsymbol{N}_{\alpha+1}$, thus such a function exists).

$$
K_{\alpha+1}=\left\{\Subset\left(f_{\alpha+1}, g\right): g \in \aleph_{\alpha+1}\{0,1\}\right\} .
$$

(c) Assume $\lambda$ is a limit ordinal and that for all $\alpha \in \lambda, K_{\alpha}$ is well defined. Let $f_{\lambda}$ be a 1-1 function on $\boldsymbol{N}_{\lambda}$ into $U_{\alpha \in \lambda} K_{\alpha}$.

$$
K_{\lambda}=\left\{\subseteq\left(f_{\lambda}, g\right): g \in \aleph_{\lambda}\{0,1\}\right\} .
$$

It should be observed that each member $\mathfrak{A}$ of any $K_{\alpha}$ is rigid, that is the only automorphism of $\mathfrak{A}$ onto $\mathfrak{A}$ is the identity.

2. Let $\tau=\{(0, n)\}$ be a similarity type where $n$ is any integer. An algebra of type $\tau$ is called an $n$-ary algebra. It follows as a direct consequence of Theorem 1 that for any positive integer $n$ and any infinite cardinal $\mathfrak{m}$ there are at least $2^{\mathfrak{m}}$ nonisomorphic algebras of power $\mathfrak{m}$ (any unary operation can be thought of as an $n$-ary operation acting on the first coordinate). Thus, for any positive integer $n$ and any infinite cardinal $\mathfrak{m}$ there are exactly $2^{\mathfrak{m}}$ nonisomorphic $n$-ary algebras with power $\mathfrak{m}$ (note that any such algebra is associated with a subset of $n+1$ tuples of its universe and there are at most $2^{\mathfrak{m}^{n+1}}=2^{\mathfrak{m}}$ such subsets).

A trivial similarity type is one whose range is $\{0\}$.

THEOREM 2. For any infinite cardinal $\mathfrak{m}$ and any nontrivial similarity type $\tau$ containing $\mathfrak{n}$ elements there are exactly $2^{\mathfrak{m} \cdot \mathfrak{n}}$ nonisomorphic algebras of type $\tau$ with power $\mathrm{m}$.

Proof. For any $\tau$-algebra $\mathfrak{A}=\langle A, \mathcal{\theta}\rangle$ of power $\mathfrak{m}, \mathcal{O}$ is uniquely associated with a member of $\mathscr{D}_{\tau}\left(\bigcup_{i \in \omega} i_{A}\{0,1\}\right)$ (i.e., we identify $n$-ary operations with certain $(n+1)$-ary relations). Hence there are at most $\left(\sum_{i \in \omega} 2^{\mathfrak{m}}\right)^{\mathfrak{n}}=2^{\mathfrak{m} \cdot \mathfrak{n}}$ different $\tau$-algebras whose universe is $A$ and hence there are at most $2^{\mathfrak{m} \cdot \mathfrak{n}}$ nonisomorphic $\tau$-algebras of power $\mathfrak{m}$.

Assume $\tau(\alpha) \neq 0$. We know that there are $2^{\mathfrak{m}}$ nonisomorphic $\tau(\alpha)$ algebras with power $\mathfrak{m}$, thus if $\mathfrak{n} \leqq \mathfrak{m}$ there are at least $2^{\mathfrak{m}}=2^{\mathfrak{m} \cdot \mathfrak{n}}$ nonisomorphic $\tau$-algebras with power $\mathfrak{m}$. If $\mathfrak{n}>\mathfrak{m}$ construct a family $L$ of $\tau$-algebras on the set $\mathfrak{m}$ by choosing $\mathcal{O}_{\alpha}$ with range $\{\alpha\}$ for all $\alpha \in \mathfrak{m}$ and $\boldsymbol{\theta}_{\alpha}$ arbitrary for all $\alpha \in \mathfrak{n} \sim \mathfrak{m}$. The family $L$ contains $2^{\mathfrak{n}}=2^{\mathfrak{m} \cdot \mathfrak{n}}$ pairwise nonisomorphic $\tau$-algebras with power $\mathfrak{m}$. In either case there are at least $2^{\mathfrak{m} \cdot \mathfrak{n}} \tau$-algebras with power $\mathfrak{m}$.

If $\tau$ is a trivial similarity type with $\mathfrak{n}$ elements and $\mathfrak{m}$ is an infinite cardinal, the argument given in Theorem 2 shows that if $\mathfrak{m}<\mathfrak{n}$ there 
are exactly $2^{\mathfrak{n}}$ nonisomorphic $\tau$-algebras with power $\mathfrak{m}$. If $\mathfrak{n} \leqq \mathfrak{m}$ there are as many nonisomorphic $\tau$-algebras with power $\mathfrak{m}$ as there are ways to partition a set with $\mathfrak{n}$ elements.

The arguments involved in the results of this section can be extended without difficulty to determine the number of isomorphism types of infinite structures whose similarity type contains both (finitary) operations and relations. We state the general result.

Suppose $\tau$ is a (general) similarity type with a total of $\mathfrak{n}$ relation and operation symbols of which $\mathfrak{n}^{\prime}$ are relation symbols and $\mathfrak{m}$ is an infinite cardinal. If $\tau$ contains at least one operation symbol of rank $\geqq 1$ or relation symbol of rank $\geqq 2$, then there are $2^{\mathfrak{m n}}$ isomorphism types of structures with power $\mathfrak{m}$ and similarity type $\tau$. On the otherhand, if all operations are 0 -ary and all relations are unary, there are $2^{\mathfrak{n}}$ isomorphism types in case $\mathfrak{n} \geqq \mathfrak{m}$ and there are $\mathfrak{m}^{\mathfrak{l}}$ isomorphism types in case $\mathfrak{n}<\mathfrak{m}$ where $\mathfrak{l}$ is the minimum of $2^{\mathfrak{n} \prime}$ and $\mathfrak{m}$.

\section{REFERENCE}

1. M. A. Harrison, Note on the number of finite algebras, J. Combinatorial Theory 1 (1966), 395-397.

VANDERBILT UNIVERSITY AND

Fisk UNIVERSITY 